\title{
Detección de inhibidores de factor viii y ix en pacientes que reciben factores de coagulación liofilizados como profilaxis o tratamiento
}

\section{Detection of factor viii and ix inhibitors in patients receiving lyophilized coagulation factors as prophylaxis or treatment}

\author{
Marcela Mardones Montanares ${ }^{1}$, Francisco Pérez Pazmiño ${ }^{1}$, Marco Pino Bedón ${ }^{1}$ \\ ${ }^{1}$ Facultad de Medicina, Pontificia Universidad Católica del Ecuador. Quito, Ecuador. \\ *mmardones382@puce.edu.ec
}

https://doi.org/10.26807/remcb.v38i2.545

Recibido 9-01-2017 Aceptado 21-06-2017

RESUMEN.- Al paciente con hemofilia se le administra como profilaxis o tratamiento el FVIII o FIX liofilizado; en ocasiones, el organismo lo reconoce como extraño y crea anticuerpos que neutralizan su efectividad. Son inhibidores dependientes del tiempo y su aparición se ve influenciado por predisposición genética. El objetivo de esta investigación fue determinar la frecuencia de los inhibidores de FVIII y FIX en 104 pacientes, de 1 a 59 años, que recibieron factores liofilizados comerciales. Se valoraron 34,6 \% pacientes con hemofilia "A" moderada; 23,08 \% severa y 13,4 $\%$ leve; $2,88 \%$ hemofilia "B" moderada. Adicionalmente se incluyeron seis pacientes $(5,77 \%)$ con Enfermedad de Von Willebrand que recibieron FVIII, en los que es probable que la efectividad del tratamiento con desmopresina no fue la ideal. El 20,19\% recibió factor VIII pero no conocía el nombre de su patología. El 40,8 \% de los resultados fue positivo para la prueba Bethesda y cinco pacientes presentaron valores mayores a cinco unidades Bethesda, es decir "altos respondedores". Se evidenció la necesidad de practicar esta prueba como prueba de rutina, en los pacientes que reciben seguido plasmas con factores liofilizados.

PALABRAS CLAVES: anticuerpos, factores de coagulación, hemofilia, inhibidores, prueba Bethesda.

\begin{abstract}
In hemophilia patients receiving factor VIII (FVIII) or factor IX (FIX), either as prophylaxis or treatment, sometimes the immune system label them as foreign bodies with consequent antibody development which reduce effectiveness. Those are time dependent inhibitors and are influenced by genetics. The aim of the study was to measure FVIII and FIX inhibitors presence in 104 patients, 1 to 59 years old, that had received commercial lyophilized factors. Moderate hemophilia "A" was identified in 34,6 \% of FVIII treatment patients, severe in 23,08 \%, and mild in $13,4 \%$. While moderate hemophilia "B" was present in 2,88 \% of cases. In addition, six Von Willebrand's disease patients $(5,77 \%)$ receiving FVIII were included likely response to desmopressin treatment was not effective. The $20,29 \%$ of FVIII receiving patients were unable to name their pathology. The $40,8 \%$ of Bethesda tests were positive, five patients showed values above 5, that label them as "high respondents". It is clear the need to perform Bethesda test as routine test in patients receiving lyophilized plasm.
\end{abstract}

KEYWORDS: antibodies, clotting factors, hemophilia, inhibitors, Bethesda test.

\section{INTRODUCCIÓN}

En el año 2004, la Federación Mundial de Hemofilia registró 17000 pacientes con hemofilia en el mundo. Se estima que en Latinoamérica existen de 1000 a 3000 pacientes con inhibidores de factores VIII (FVIII) y IX; la hemofilia severa es la que se encuentra en el mayor número de estos pacientes. En el año 2010, mediante el último censo del Ministerio de Salud Pública (MSP) y la Fundación Hemofílica Ecuatoriana (FUNDHEC) se registraron 734 pacientes con hemofilia. Esta enfermedad crónica se conoce a nivel mundial, sin embargo, en Ecuador solamente a partir del año 2006, se impulsa el estu- 
dio de la prevalencia de pacientes con hemofilia, la detección de personas portadoras de hemofilia y el adiestramiento en la práctica de técnicas de laboratorio que mejorarán la calidad de vida del paciente. Entre las primeras pruebas realizadas, se determinó la presencia de anticuerpos generados a partir de la transfusión con crioprecipitados y plasmas frescos congelados que fueron los primeros productos terapéuticos utilizados en pacientes con hemofilia y otras coagulopatías y que dejaron definitivamente de ser utilizados en el año 2008, por el riesgo que generaba a la salud el uso constante de estos productos, dando paso al uso de plasmas liofilizados.

El sistema inmune tiene un papel importante en la formación de los inhibidores contra el factor VIII o IX, además de la condición hereditaria presentada en cada paciente. Los inhibidores son anticuerpos del tipo IgG que se dirigen contra los dominios de la molécula de FVIII: A1, C1 y C2. Una persona con hemofilia A puede crear inhibidores después de la administración del tratamiento para remplazar el factor carente. Veinticinco por ciento de las personas desarrollan inhibidores durante las primeras 75 exposiciones a los concentrados de factor, el mayor riesgo de desarrollar inhibidores se encuentra entre las primeras 10 a 20 dosis de tratamiento, lo que significa que se presenta principalmente en niños con hemofilia severa, aunque estos anticuerpos pueden aparecer y desaparecer después de recibir el tratamiento (Castillo 2012)

Los inhibidores de factor IX pueden ocasionar reacciones alérgicas y anafilácticas que no son producidas en hemofilia A. La incidencia de hemofilia B es menor que la de hemofilia A y se relaciona con la presencia baja de inhibidores; sin embargo, su aparición revela títulos altos, cuando se presenta positivo para inhibidores. Los pacientes con hemofilia B con deleciones o reorganizaciones en el gen de factor IX, tienen riesgos de desarrollar inhibidores en $50 \%$ de los casos, mientras que aquellos que tienen mutaciones sin sentido tienen riesgo de $20 \%$. No se han encontrado mutaciones puntuales o cambio de sentido en esta hemofilia (Castaman et al. 2013).

En Ecuador, las pruebas utilizadas para detectar inhibidores están basadas en pruebas coagulométricas. La prueba Bethesda, por ejemplo, se determina en equipos automatizados o semiautomatizados, Actualmente esta prueba se realiza en pacientes que generan anticuerpos después de recibir la terapia de reemplazo por lo tanto no mejorará el sangrado en el paciente después del tratamiento.
Para mejorar la sensibilidad de la prueba se debería realizar una modificación a la prueba Bethesda, llamada modificación de Nijmegen (Verbruggen 1995). Esta corrección permitirá que, al bufferar el plasma control con imidazol, incremente la sensibilidad y especificidad de la prueba. Las variaciones de actividad de los inhibidores en el plasma pueden influir en la actividad del inhibidor medido (FMH 2012).

La presencia de inhibidores se encuentra con frecuencia en pacientes con hemofilia $\mathrm{A}$ y es raro o no se enceuntra en aquellos con hemofilia B (Leissinger 2016). Cuando se detecta un anticuerpo, generalmente se lo clasifica como de "alta o baja respuesta", lo cual depende de la reacción inmunológica después de repetidas exposiciones al factor administrado. En la hemofilia A, los inhibidores de mayor frecuencia que anteceden genéticamente son pequeñas deleciones, inserciones, inversión del intrón 22 y mutaciones sin sentido (Radic 2010).

En los pacientes positivos para inhibidores se encuentran dos tipos de resultados: a) los de baja respuesta, quienes poseen niveles de inhibidores $<5$ $\mathrm{UB} / \mathrm{mL}$ (unidades Bethesda por mililitro), no desarrollan incremento en los niveles de inhibidores luego de exposiciones al factor VIII o IX y su respuesta inhibitoria será más débil a los concentrados de factor; b) los de alta respuesta, quienes poseen títulos de inhibidores $>5 \mathrm{UB} / \mathrm{mL}$, encontrado en una ocasión y con la exposición repetida del concentrado desencadena la formación de más inhibidores (FMH 2012).

El tratamiento en estos pacientes consiste en eliminar los anticuerpos encontrados, no solo con inmunosupresión, sino con la inmunotolerancia, que se presenta como la mejor opción terapéutica. Esta técnica consiste en entrenar al organismo a recibir dosis frecuentes de factor VIII o IX durante algún tiempo y que este sea reconocido por el sistema inmune como familiar (Kreus y Escuriola 2013).

El objetivo de esta investigación fue determinar la frecuencia de los inhibidores de factor VIII o IX en pacientes que reciben plasmas liofilizados como profilaxis o tratamiento.

\section{MATERIALES Y MÉTODOS}

El estudio fue descriptivo y transversal; las muestras de sangre fueron de 104 pacientes con hemofilia A o B, infundidos plasmas liofilizados de FVIII o IX. 
Las muestras de sangre fueron tomadas de pacientes que residen en las ciudades de Ambato $(\mathrm{N}=33)$, Cuenca; Azogues $(\mathrm{N}=7)$, Guayaquil $(\mathrm{N}=33)$, Quito; Ibarra $(\mathrm{N}=25)$ y Santo Domingo de los Tsáchilas $(\mathrm{N}=6)$. . Estos pacientes están registrados en la Fundación Hemofílica Ecuatoriana (FUNDHEC).

Al llamado también acudieron pacientes con Enfermedad con Von Willebrand que recibieron el mismo tratamiento. Todos fueron pacientes que estuvieron expuestos al factor VIII o IX como mínimo 72 horas, hubo también pacientes que no recibieron fac-
El procesamiento de la prueba Bethesda se realizó según el protocolo de Kitchen et al. (2010). Esta prueba fue realizada para las muestras de pacientes en los que no era previsible el inhibidor, se realizó una dilución 1:1 de la muestra de los pacientes y un pool normal. Como prueba control, se añadió en partes iguales el pool normal y el buffer de Owren, se incubó por dos horas y se realizó el ensayo de factor VIII. Para la prueba de Factor IX, se incubó 10 minutos y se realizó la lectura de manera igual a la anterior prueba.

Tabla 1. Distribución de la hemofilia según su gravedad y de la enfermedad Von Willebrand

\begin{tabular}{lcc}
\hline \multicolumn{1}{c}{ Tipo de hemofilia } & Frecuencia & Porcentaje \\
\hline Desconocido & 21 & $20,19 \%$ \\
Hemofilia A moderada & 36 & $34,62 \%$ \\
Hemofilia A leve & 14 & $13,46 \%$ \\
Hemofilia A severa & 24 & $23,08 \%$ \\
Hemofilia B moderada & 3 & $2,88 \%$ \\
Enfermedad von Willebrand (EVW) $\dagger$ & 6 & $5,77 \%$ \\
TOTAL & 104 & $100,00 \%$ \\
\hline
\end{tabular}

$\dagger$ Al llamado asistieron 6 pacientes con EVW, quienes recibieron factor VIII liofilizado

tor hace seis meses. Fueron valorados 21 pacientes que recibieron factor VIII, pero no tenían la seguridad de responder sobre el grado de hemofilia A que padece. En la recolección de los datos se informó de ocho pacientes que nunca recibieron factor VIII o IX, se les procesó como controles normales (Tabla 1). A los pacientes con hemofilia se les realizó hace un año el control de presencia de inhibidores. Ninguno de los pacientes que acudieron a la toma de muestra llegó con sangrados. La participación fue voluntaria, previa a la firma del consentimiento informado. Esta investigación fue realizada en el período de un año.

Se determinó la frecuencia de anticuerpos inhibidores de factor VIII y IX en pacientes hemofílicos, sin que exista una hemorragia o una falta de respuesta a la terapéutica. Para ello se empleó una metodología semiautomática, utilizando el equipo Start4 de marca Stago. Para la calibración de curva de factor VIII o IX residual, se realizaron diluciones de $1 / 10,1 / 20,1 / 50,1 / 250$ con buffer de Owren y Unicalibrador, los tubos fueron tapados con parafilm para la incubación durante dos horas y se realizó la lectura. Todas las pruebas se desarrollaron en el laboratorio de la Facultad de Medicina de la Pontificia Universidad Católica del Ecuador (PUCE).
En muestras de pacientes en los que fue previsible el inhibidor, se prepararon diluciones de $1 / 10$ al 1/512 con buffer de Owren y a cada dilución se colocó una cantidad estandarizada de factor VIII dado en un pool normal. Se incubó por dos horas y se midió la cantidad de factor VIII residual.

Se calculó el inhibidor a partir de una gráfica establecida y mediante tablas que establecen la cantidad de factor VIII residual versus Unidades Bethesda (UB) encontradas. Se deben determinar los niveles de inhibidores luego de mínimo 48 a 72 horas de la última administración de factores(Tabla 2).

Para el análisis estadístico de los resultados se utilizó el programa EpiInfo 7. Los resultados se presentan en tablas de frecuencias para establecer la presencia o no del inhibidor en los pacientes, la relación entre la prueba Bethesda y la clasificación en altos y bajos respondedores. Además, la asociación entre la concentración del inhibidor y la severidad de la hemofilia se analizó usando la prueba de Chi Cuadrado $\left(\mathrm{X}^{2}\right)$, asumiendo un nivel de significancia de $\mathrm{p}<0,05$.

\section{RESULTADOS}

Cuarenta y dos pacientes fueron positivos en la 
Tabla 2. Distribución de pacientes según tiempo de haberse admintrado factor VIII o IX

\begin{tabular}{lcc}
\hline \multicolumn{1}{c}{ Tratamiento } & Frecuencia & Porcentaje \\
\hline Menos de 1 mes & 41 & $41,30 \%$ \\
Nunca ${ }^{\dagger}$ & 8 & $5,43 \%$ \\
Más de 1 mes & 55 & $53,26 \%$ \\
TOTAL & 104 & $100,00 \%$ \\
\hline
\end{tabular}

$\dagger$ Los pacientes que asistieron al llamado e indicaron que nunca recibieron factores liofilizados fueron procesados como controles negativos. Los inhibidores son dependientes del tiempo, se esperaría que los pacientes con menos de un mes presenten positividad en la prueba Bethesda

prueba Bethesda (40,38\%), de estos, cinco pacientes fueron altos respondedores: uno de la ciudad de Cuenca, tres de Guayaquil y uno de Santo Domingo (Tabla 3). No existe evidencia que las diferentes marcas de casas comerciales de plasmas liofilizados utilizados en el tratamiento sean los que generen esta clase de inhibidores, pero lo que sí se sugiere, de manera urgente, es el cambio de tratamiento de los pacientes, con "bypassing" recombinantes o concentrados de complejos protrombínico. Para esto, el MSP debe mantener dosis de reserva que minimicen el impacto que afecta la calidad de vida del paciente con hemofilia y con inhibidores de alta

Tabla 3. Análisis de la Pruea Bethesda y la ciudad de residencia

\begin{tabular}{lccc}
\hline \multicolumn{1}{c}{ Ciudad } & Negativo & Positivo & TOTAL \\
\hline Ambato & 15 & 18 & 33 \\
Cuenca/Azogues $\dagger$ & 6 & 1 & 7 \\
Guayaquil & 22 & 11 & 33 \\
Quito/Ibarra $\dagger$ & 14 & 11 & 25 \\
Santo Domingo & 5 & 1 & 6 \\
TOTAL & 62 & 42 & 104 \\
\hline Chi-cuadrado+ 4,274; df 4; Probabilidad 0,37 & \\
+Se espera valores $<$ 5 para que el resultado sea significativo & \\
$\dagger$ una muestra de Azogues v una de Ibarra &
\end{tabular}

respuesta.

No existen diferencias significativas en la distribución de pacientes con resultados negativos según la ciudad donde residen (Tabla 3 ). Cada provincia debe asegurarse de formar un núcleo de pacientes para fortalecer el trabajo a nivel provincial y determinar cuál es la distribución de pacientes con inhibidores según las provincias, de esa manera el MSP sabrá que medicamentos deben ser redistribuidos y no centralizarse en la capital o solamente en las grandes ciudades.

No se detectaron diferencias estadísticamente significativas en la distribución de los resultados de las pruebas de detección de inhibidores cuando se compara por el tipo de hemofilia declarado por los pacientes (Tabla 4). Esto nos indica que la presencia de inhibidores puede estar presente sin distinción en cualquiera de los tipos de hemofilia y Von Willebrand.

El promedio de edad en los pacientes atendidos fue de veinte y un años, lo que indica que los pacientes que participaron en el estudio fueron la mayoría adolescentes, este grupo de pacientes vulnerables, requiere de un mejor sistema de monitoreo de inhibidores que les permitirá tener mejores expectativas de vida con menos secuelas de la enfermedad.

Tabla 4. Análisis del tipo de hemofilia y la prueba Bethesda

\begin{tabular}{lccc}
\hline \multicolumn{1}{c}{ Tipo de hemofilia } & Negativo & Positivo & TOTAL \\
\hline Desconocido ${ }^{\dagger}$ & 12 & 9 & 21 \\
Hemofilia A moderada & 19 & 17 & 36 \\
Hemofilia A leve & 11 & 3 & 14 \\
Hemofilia A severa & 12 & 12 & 24 \\
Hemofilia B moderada & 3 & 0 & 3 \\
Enf. Von Willebrand & 5 & 1 & 6 \\
TOTAL & 62 & 42 & 104 \\
\hline
\end{tabular}

Chi-cuadrado+ 3,677; df 5; Probabilidad 0,597 (Yates)

+ Se esperan valores $<5$ para que el resultado sea significativo.

$\dagger$ Pacientes que se infundían factor VIII pero no tenían el conocimiento de la

\section{DISCUSIÓN}

La hemofilia es una coagulopatía que tiene grandes implicaciones en la vida del paciente, como en el caso de personas que reciben factores de la coagulación liofilizados como tratamiento o profilaxis, y en donde pueden aparecer anticuerpos o inhibidores de FVIII o IX. Según lo indicado en la Guía de Tratamiento en Hemofilia (Fundación de la hemofilia 2011) la cuantificación de inhibidores debe aplicarse rutinariamente una vez por año y dos veces al año, sí el paciente es menor de cinco años.

Los pacientes que participaron en esta investigación solo son atendidos al presentar sintomatología y no tener respuesta al tratamiento. En el reporte referido por Connie Miller (2015) se recomienda a todos los pacientes realizarse la prueba de inhibidores cada año, y llevar un monitoreo más seguido a aquellos que ya presentan inhibidores, esto permitirá la selección de una terapia adecuada, además de un mejor monitoreo y la erradicación del inhibidor.

El estudio evaluó 104 pacientes diagnosticados con hemofilia A o B y EVW, residentes en siete ciudades de diferentes provincias del Ecuador y que voluntariamente, se sometieron a la prueba. Varias provincias del país carecen de estadísticas y de pruebas para diagnosticar la enfermedad. A nivel mundial se 
indica que la hemofilia A se presenta entre 1:5000 a 1:10 000 nacidos vivos, mientras que la hemofilia $\mathrm{B}$ entre 1:30 000 nacidos vivos (Guía práctica Clínica 2016) (Stonebraker et al. 2011). En el presente estudio las diferencias según la residencia de los pacientes en cuanto a si se identifican resultados positivos para la prueba Bethesda no mostraron diferencias significativas.

Se detectó que los pacientes con hemofilia moderada presentaron el mayor porcentaje de inhibidores con $34,62 \%$, seguido de la hemofilia severa con $23,8 \%$ y la hemofilia leve con $13,46 \%$, pruebas realizadas por métodos coagulométricos, según Castillo (2014) en su estudio con 229 pacientes en cinco provincias de Cuba, detectó 56,3\% de pacientes con hemofilia severa, $24 \%$ moderada y 19,7 $\%$ leve lo que no concuerda con lo obtenido en esta investigación. La prevalencia a nivel mundial menciona a la hemofilia severa como la que se encuentra en mayor proporción (Witmer y Young 2013).

Según Leissinger (2016), la prevalencia de inhibidores en hemofilia A severa es del $30 \%$ y en hemofilia B, alrededor de $3 \%$. La mayoría se presenta en edades tempranas de vida (edad media de 1,7 - 3,3 años) con mínimo de $10-20$ exposiciones al factor VIII y la presencia de inhibidor de FIX no es común. Se debe indicar que los inhibidores también están presentes en la edad adulta. En el estudio realizado en 104 pacientes, cuyas edades incluía mayoritariamente niños y adolescentes, se obtuvieron resultados positivos para la prueba Bethesda: hemofilia A moderada 17 pacientes, hemofilia A severa 12 , hemofilia A leve 3, EVW 1 paciente, lo que evidencia en total $40,38 \%$ son positivos para la prueba Bethesda, de ellos 4,8\% fueron pacientes respondedores altos, tres niños; el 35,58\% de los pacientes presentaron títulos bajos de anticuerpos. Se considera que la presencia de estos inhibidores es la más seria complicación asociada al tratamiento con hemofilia, son pacientes que fácilmente pueden presentar hemorragias incontrolables acortando su calidad de vida, además de aumentar el riesgo de mortalidad. En los pacientes que presentaron títulos bajos se considera el aumento de la terapia de remplazo. La prueba Bethesda, en este estudio, no mostró diferencias estadísticamente significativas en relación con el tipo de hemofilia reportado por los pacientes.

La predisposición genética es otro factor que induce a la formación de inhibidores debido a diferentes mutaciones, en especial la inversión del intrón 22 del gen Factor VIII (F8), detectada con frecuencia en hemofilia A, además de encontrarse en familias con un historial de presencia de anticuerpos contra el factor VIII o IX (Leissinger 2016). Esto permitirá dar una guía en la investigación con nuevas técnicas moleculares e integradas como pruebas diagnósticas habituales para los pacientes con hemofilia.

El desarrollo de la técnica para detectar inhibidores se realizó a base de la prueba estandarizada Bethesda, creada por Kasper en 1975 (Kasper 2004) y utilizada para cuantificar los inhibidores presentes. En este estudio se detectó el 4,8\% de pacientes de "alta respuesta", no asociado al porcentaje de pacientes positivos para la prueba Bethesda. Por otra parte este dato permite guiar al médico en el tipo de tratamiento que requiere y su adecuada monitorización.

Los pacientes con hemofilia B resultaron negativos para inhibidores. La detección de los inhibidores es baja relacionada con la hemofilia A. En su artículo Nassef (2016) resalta la incidencia de inhibidores en hemofilia B del 1-5\% y su presencia caracteriza al paciente con hemofilia moderada tipo $\mathrm{B}$, con ocasionales sangrados que requieren de la terapia de remplazo con factores liofilizados. En los pacientes con hemofilia A el 4,8\% presentaron valores mayores a 5 UB ( $>5$ UB). Son pacientes llamados "altos respondedores" y se encuentra hemofilia severa y moderada. Para estos resultados, existen procedimientos que proporcionan tolerancia inmunológica al paciente y que permiten que poco a poco los anticuerpos lleguen a desaparecer. El $35,58 \%$ de pacientes presentaron títulos bajos, de ellos $15,34 \%$, presentan valores menores 0,6 UB $(<5$ UB) incluido el paciente con EVW que presentó $0,3 \mathrm{UB}$, título de anticuerpos que no significa mayor riesgo para el paciente, denominados "bajos respondedores", pero que obligan a un monitoreo continuo cada tres meses, Lo que coincide con la investigación realizada por Miller et al. (2015) que indica que la población afectada con inhibidores no presentó una atención previa con el clínico y que la presencia de títulos altos o bajos en inhibidores deben monitorearse, pues no se conoce si habrá un aumento, disminución o será transitoria la cantidad de inhibidores, queda realizar una nueva valoración de anticuerpos con otra cohorte y verificar si son anticuerpos transitorios o permanentes.

El detectar tempranamente los inhibidores mejorará la respuesta a la terapia, aun si los inhibidores están en títulos bajos. Su presencia disminuye la calidad de vida del paciente y representa un impacto alto económicamente, debido al incremento en el costo del tratamiento. La detección oportuna y el tratamiento adecuado en el paciente con inhibidores 
mejorarán con la aplicación de técnicas y análisis de laboratorio apropiados, y al tener los productos plasmáticos específicos para cada patología y recombinantes adecuados.

\section{REFERENCIAS BIBLIOGRÁFICAS}

Castaman G, Bonetti E, Messina M, Morfini M, Rocino A, Scaraggi FA, Tagariello G. (2013). Inhibitors in haemophilia B: the Italian experience. Haemophilia. [citado enero 2015]; 2013(19): 686690. Recuperado de: file:///K:/disco\%20 extra\%C3\%ADble\%20flash\%20azul/ proyecto\%20universidad/Bibliograf\%C3\%ADa\%20inhibidores/castaman2013. pdf doi: 10.1111/hae. 12158

Castillo D. (2012). Hemofilia: aspectos históricos y genéticos. Revista Cubana de Hematología, Inmunología y Hemoterapia 28(1): 22-23.

Castillo D. (2014). Prevalencia de hemofilia en seis provincias cubanas. Revista Cubana de Hematología, Inmunnologìa y Hemoterapia. $30(2): 155-161$.

[FMH] Federación Mundial de Hemofilia (Canadá). (2012). Guía para el tratamiento de la Hemofilia. Toronto: Blackwell Plublishing.

Fundación de la Hemofilia. (2011). Guía de Tratamiento de la hemofilia. Buenos Aires: Fundación de la Hemofilia

[MSP] Ministerio de Salud Pública. (2016). Diagnóstico y tratamiento de la hemofilia congénita. Guía de Práctica Clínica. Quito: El telégrafo.

Kasper C. (2004). Diagnóstico y tratamiento de inhibidores de factor VIII y IX. Tratamiento de la hemofilia $34.13 \mathrm{p}$

Kreus W and Escuriola C. (2013). Inhibitors in patients with haemophilia A. Thrombosis Research.[citado marzo 2015]; 134(1): 2-5. Recuperado de:http://dx.doi. org/10.1016/ j.thromres.2013.10.016.

Leissinger C. (2016). Advances in the clinical management of inhibitors in hemophilia A and B. Seminars in Hematology 53:2027.
Miller C. (2015). Improving the performance of factor VIII inhibitors test in hemophilia A. Thrombosis Research 136:1047-1048.

Miller C, Rice A, Boylan B, Payne A, Kelly F, Escobar M, Gill J, Leissinger C, Soucie J. (2015). Characteristics of hemophilia patients with factor VIII inhibitors detected by prospective screening. Am Journal Hematology. 90(10): 871-876. Recuperado de:https://www.ncbi.nlm.nih.gov/pmc/ articles/PMC4642843/pdf/nihms733784. pdf. doi: 10.1002/ajh. 24104.

Nassef M, Sheenan J. (2016). New develoments in the management of moderate-to-severe hemophilia B. Journal of blood Medicine 7:27-38. Recuperado de:https:// doi. org/10.2147/JBM. S81520

Radic C. (2010). Genética molecular de la hemofilia: caracterización de mutaciones en hemofilia $\mathrm{B}$, expresión de hemofilia en mujeres y desarrollo de nuevos métodos de análisis de inversiones. Tesis Doctoral. $131 \mathrm{p}$.

Stonebraker J, Bolton P, Soucie J, Walker I, Brooker M. (2011). Federación mundial de hemophilia: Estudio de las variaciones en los informes de prevalencia de la hemofilia alrededor del mundo. Haemophilia 16: $1-32$.

Witmer Ch, Young G. (2013). Factor VIII inhibitors in hemophilia A: rationale and latest evidence. Therapeutic Advances in Hemato$\operatorname{logy} 4(1): 59-72$. 